\title{
Synthesis and physical characterization of carbon nanotubes coated by conducting polypyrrole
}

\begin{abstract}
This study describes the preparation of polypyrrole multiwall carbon nanotube (PPy/MWNT) composites by in situ chemical oxidative polymerization. Various ratios of functionalized MWNTs are dispersed in the water, and PPy are then synthesized via in-situ chemical oxidative polymerization on the surface of the carbon nanotubes. The morphology of the resulting complex nanotubes (MWNT-PPY) was characterized by field-emission scanning electron microscopy (FESEM). The conductivity of each composite showed a maximum in the temperature scale of $120 \ddot{\mathrm{i}} 160{ }^{\circ} \mathrm{C}$ and then decreased dramatically with the increase of temperature. The resultant PPy/MWNT nanotubes enhanced electrical conductivity and thermal stability of nanocomposite compared to PPy which was strongly influenced by the feed ratio of pyrrole to MWNTs.
\end{abstract}

Keyword: Carbon nanotube; Conducting polymer; Electrical conductivity; Thermal stability 